\title{
Modelling high-temperature creep of anisotropic material
}

\author{
R N GHOSH \\ National Metallurgical Laboratory, Jamshedpur 831 007, India
}

\begin{abstract}
Cast single-crystal superalloys are being used as turbine blades in modern aeroengines with $\langle 001\rangle$ axis oriented along the direction of principal stress. This allows use of higher operating temperature with associated improvement in efficiency. Although $\langle 001\rangle$ is the natural direction of crystal growth in these alloys it is often not possible to attain perfect alignment. Consequently, besides time-dependent deformation this would induce a change in cross-sectional shape and crystal orientation of the blade as a result of service exposure. Therefore in order to exploit their full potential it is necessary to develop a predictive system which could model effect of crystallographic anisotropy on hightemperature creep deformation of such materials. The paper reviews the recent developments in this direction and suggests that modelling not only helps in identifying the dominant mechanisms of deformation but also suggests simple experiments to verify them.
\end{abstract}

Keywords. Superalloy; single crystal; octahedral/cube slip; deformation mechanism; orientation change; SRR99.

\section{Introduction}

The development of single-crystal superalloys and their introduction as turbine blades in aeroengines have been the major achievements in gas-turbine technology during the last decade. This also provides an example of how the anisotropic behaviour of materials could be exploited to improve the performance (or efficiency) of the engine by raising its safe operating temperature. It is reported that the use of single-crystal superalloys such as PWA 1480 , SRR99 and CMSX2 offers at least $50^{\circ} \mathrm{C}$ higher temperature capabilities than those of conventionally cast or directionally solidified alloys (e.g. Mar M 200, Mar M 002). However, in order to exploit their full potential through the use of advanced computer-based design procedures it is necessary to characterize fully their anisotropic behaviour.

There have been several experimental studies on anisotropic creep behaviour of single-crystal superalloys. There is some controversy as to whether $\langle 001\rangle$ or $\langle 111\rangle$ crystal orientations are strongest. Caron et al (1988) have shown that $\gamma^{\prime}$ size and morphology can have a profound effect on both the magnitude and nature of this anisotropy. The mechanisms of deformation in $\gamma^{\prime}$-strengthened superalloys can be very complex depending on the distribution of $\gamma^{\prime}$ and the conditions of deformation. Evidence of both octahedral slip having either $\langle 101\rangle$ or $\langle 211\rangle$ Burgers vectors and cube slip have been reported (Kear et al 1967; Caron et al 1988) after tensile deformation at $750-980^{\circ} \mathrm{C}$. Clearly the extent of anisotropy will depend on the number and the type of operative slip systems. Ghosh et al (1990) proposed a model for simulating the creep behaviour of anisotropic material assuming this to be determined by shear deformation on various slip planes. An analysis of the creep curves of SRR99 over a range of stresses and temperatures using this approach indicates that creep in these alloys is controlled by both octahedral and cube slip. The present work describes the basic principles of modelling creep of single crystals and suggests simple experimental methods of identifying the operative slip system. 


\section{Physical model}

A series of papers from National Physical Laboratory, UK, and Cambridge University have described a model of creep deformation of isotropic engineering alloys based on the formalism of continuum damage mechanics. The most novel aspect of this approach is the replacement of the empirical equations of the type introduced by Kachanov (1958), Robonotov (1969) and Leckie and Hayhurst (1977) with explicit equations that are fully consistent with current understanding of deformation mechanism in engineering materials. The model has been incorporated in a software package called CRISPEN. Dyson and McLean (1990) recently reviewed its current status in describing creep behaviour of engineering alloys particularly those exhibiting substantial tertiary creep. Dyson and Ashby (1984) identified three broad categories of damage that can make significant contributions to tertiary creep. They are: (i) loss of external section due to geometrical changes leading to a change in stress under constant loading condition $\left(\omega_{1}\right)$, (ii) loss of internal load-bearing section due to cavitation or cracking which occurs commonly at grain boundaries $\left(\omega_{2}\right)$, (iii) changes in the material microstructure (e.g. dislocation density, particle size) that reduce the strength of the material $\left(\omega_{3}\right)$.

The damage parameter $\omega_{i}$ can be defined in a number of ways. Assuming that $\omega_{i}$ is a linear function of strain it can be shown that creep rate $\dot{\varepsilon}$ is an exponential function of $\omega_{1}$ and $\omega_{2}$ (Dyson and Gibbons 1987; Dyson 1988). However, the effect of $\omega_{3}$ has been represented as either a linear or an exponential dependence on creep rate (Dyson and McLean 1990).

In general the overall tertiary creep behaviour of superalloys should be determined by each of the above damage mechanisms. However, the situation is somewhat simpler in the case of single-crystal material. Since there is no grain boundary the effect of cavitation could be ignored $\left(\omega_{2}=0\right)$. Similarly, if one were to look at constant stress creep data where the effect of decreasing cross-sectional area is compensated by proportionate load reduction, it is not necessary to consider the influence of $\omega_{1}$. Curtis et al (1993) analysed the constant stress-creep curves of SRR99 having $\langle 001\rangle$ orientations using the isotropic creep model. It has been shown that beyond $750^{\circ} \mathrm{C}$ creep behaviour of the alloy can be adequately described by the linear damage model. Consequently the tertiary creep behaviour of single-crystal superalloys of a given orientation could be represented as

$$
\begin{aligned}
& \stackrel{\circ}{\varepsilon}=\dot{\varepsilon}_{i}(1+\omega), \\
& \stackrel{\circ}{\omega}=C^{\circ},
\end{aligned}
$$

where $\stackrel{\varepsilon}{i}_{i}$ and $C$ are material constants and $\omega_{3}$ has been redesignated as $\omega$.

Although this type of description is adequate to interpret and analyse creep curves of single-crystal superalloys having a symmetric tensile axis it cannot be applied to crystals of any arbitrary orientation. This is because deformation in a single crystal takes place through glide on specific planes along specific directions. As a result a nonsymmetric tensile axis would tend to rotate about a specific direction. An appropriate model is therefore expected to predict not only the creep strain but also the amount of rotation. Ghosh et al (1990) have given a detailed account of how the model described in (1) could be reformulated in terms of shear strains on a specific slip plane and direction. This in principle would allow prediction of crystal rotation. 
In order to appreciate its full implication it is worth reexamining the basis of such a model.

The equation set (1) could be recast as follows to obtain an estimate of the magnitude of shear strain $\gamma^{K}$ on the $K$ th slip system:

$$
\begin{aligned}
& \dot{\gamma}^{K}=\dot{\gamma}_{i}^{K}\left(1+\omega^{K}\right), \\
& \dot{\omega}^{K}=C^{K} \dot{\gamma}^{K},
\end{aligned}
$$

where $\gamma_{i}^{K}$ and $C^{K}$ represent material parameters describing evolution of shear strain on the $K$ th slip system and $\omega^{K}$ is the accumulated structural damage on the specific slip system. Solution of the above equation set gives us the magnitude of accumulated shear strain on a specific slip system. In order to model the overall deformation one needs to solve similar equations for all possible slip systems. The total macroscopic displacement $\left(e_{i j}\right)$ arising out of slip deformation (or simple shear) taking place on a set of $N$ slip systems is given by

$$
e_{i j}=\sum_{K=1}^{N} \gamma^{K} b_{i}^{K} n_{j}^{K}
$$

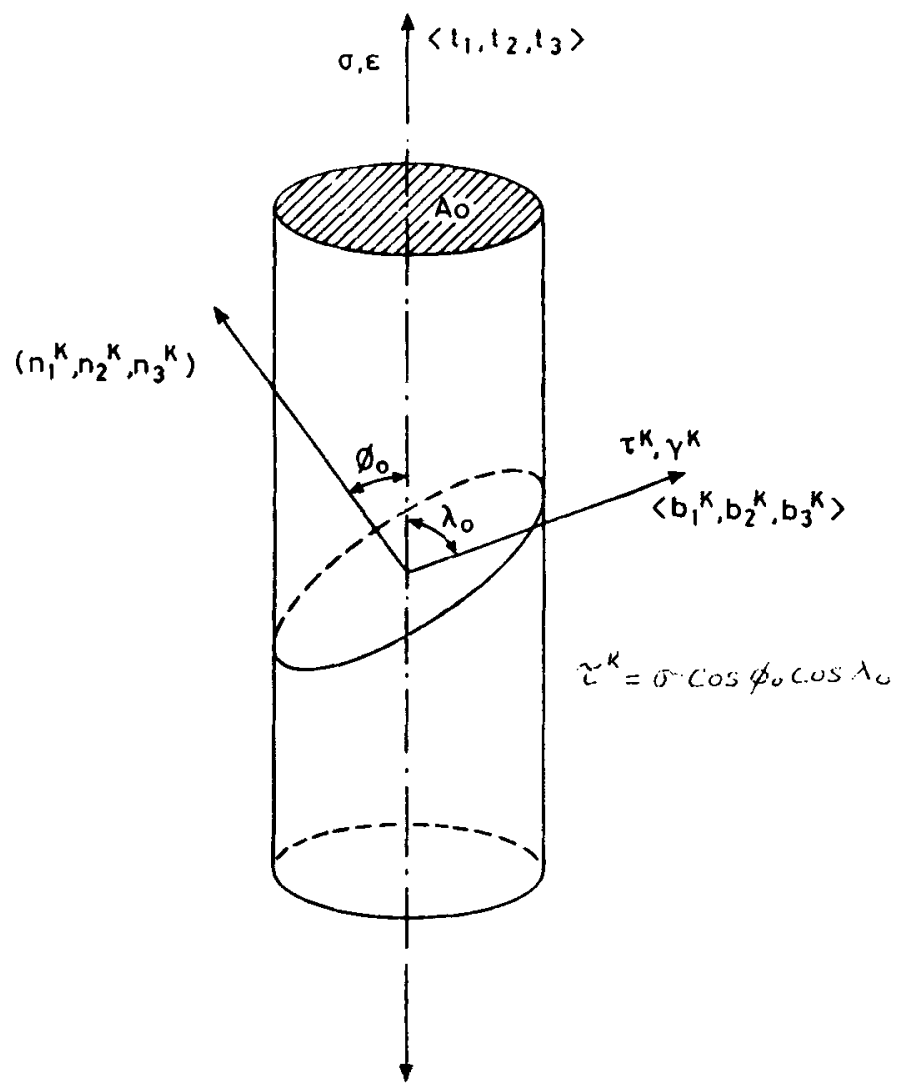

Figure 1. A schematic diagram showing the relationship of applied stress $\sigma$ and tensile strain $\varepsilon$ with slip plane $\left(n_{1}^{K}, n_{2}^{K}, n_{3}^{K}\right)$, slip direction $\left[b_{1}^{K}, b_{2}^{K}, b_{3}^{K}\right]$, resolved shear stress $\tau^{K}$ and shear strain $\gamma^{K}$. 


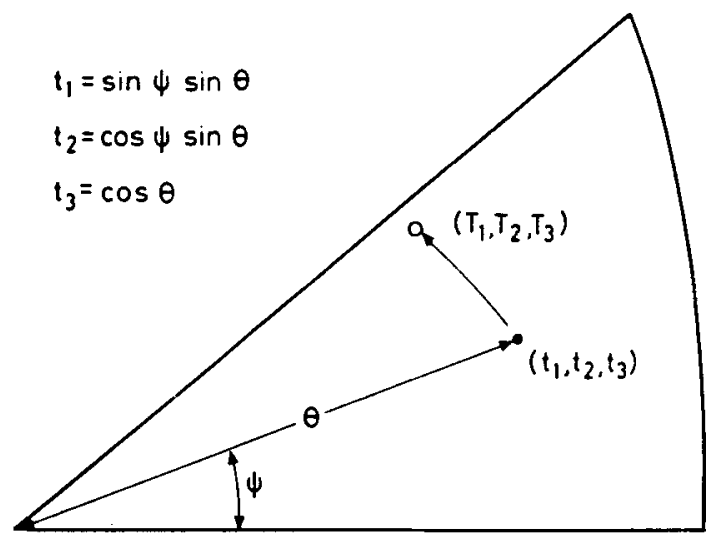

Figure 2. Schematic diagram showing the orientation relationship of an arbitrary tensile axis with various crystal directions before and after deformation.

where $\left[b_{1}^{K}, b_{2}^{K}, b_{3}^{K}\right]$ represents unit vector for the slip direction and $\left(n_{1}^{K}, n_{2}^{K}, n_{2}^{K}\right)$ is the unit vector representing slip plane normal. Figure 1 gives a schematic representation of the orientation of the loading axis with respect to the slip plane normal and the slip direction. The angular location of the tensile axis $\left\langle t_{1}, t_{2}, t_{3}\right\rangle$ has been shown with respect to three crystallographic axes of high symmetry, viz. $\langle 001\rangle\langle 011\rangle$ and $\langle 111\rangle$, on a stereographic triangle in figure 2 . As a result of slip deformation the orientation of the tensile axis is likely to change. If $\left\langle T_{1}, T_{2}, T_{3}\right\rangle$ is the vector representing the tensile axis after slip, then

$$
T_{i}=\left(e_{i j}+\delta_{i j}\right) t_{j}
$$

where $\delta_{i j}=1$ for $i=j$ and $\delta_{i j}=0$ for $i \neq j$.

The repeated subscript $j$ in (4) represents summation over $j$ from 1 to 3 . Similarly any transverse direction in the crystal such as $\left\langle x_{1}, x_{2}, x_{3}\right\rangle$ will change into $\left\langle X_{1}, X_{2}, X_{3}\right\rangle$ and the relationship between the two is given by

$$
X_{i}=\left(e_{i j}+\delta_{i j}\right) x_{j}
$$

Consequently the longitudinal $(\varepsilon)$ and transverse $\left(\varepsilon_{\mathrm{tr}}\right)$ strain could be calculated by

$$
\begin{aligned}
\varepsilon & =(\bar{T}-\bar{t}) / \bar{t}, \\
\varepsilon_{\mathrm{tr}} & =(\bar{X}-\bar{x}) / \bar{x},
\end{aligned}
$$

where $\bar{X}, \bar{T}, \bar{x}$ and $\bar{t}$ represent magnitude of the respective vectors. The amount of rotation and the axis of rotation is also described by (4). The antisymmetric part of the matrix $e_{i j}$ represents the rotation matrix $\left(\omega_{i j}\right)$ of the crystal. This is given by

$$
\omega_{i j}=\left(e_{i j}-e_{j i}\right) / 2 \text {. }
$$

The magnitude $/ \omega /$ and the axis of rotation are given by

$$
\begin{aligned}
& |\omega|=\sqrt{\omega_{12}^{2}+\omega_{23}^{2}+\omega_{31}^{2}}, \\
& \text { Rotation axis }=\frac{\omega_{23}}{|\omega|}, \frac{\omega_{31}}{|\omega|}, \frac{\omega_{12}}{|\omega|} .
\end{aligned}
$$


Equations (2) to (10) describe completely the deformation behaviour of a single crystal at constant stress and temperature. In order to extend the model to allow interpolation or extrapolation over various stress and temperature ranges one needs to establish stress and temperature dependence of the material parameters $\gamma_{i}^{K}$ and $C^{K}$. The procedure for establishing this has been discussed by Ghosh et al (1990). In subsequent part of the paper expressions used by Ghosh et al (1990) and the numerical values of these constants reported in table 3 of their paper have been used.

\section{Results and discussion}

Table 1 provides an illustrative calculation for the construction of displacement matrix of a single crystal with [001] as the tensile axis and $\{111\}\langle 1 \overline{1} 0\rangle$ as the slip system. For such a case among 12 possible combinations of slip planes and slip directions eight have identical magnitude of resolved shear stress and the remaining four have no resolved shear stress component at all. Therefore eight of the 12 octahedral slip systems will contribute equally to the total creep strain at any instant of time. In this case $\gamma$ represents the magnitude of shear strain on individual slip system whereas $\sigma / \sqrt{6}$ is the magnitude of resolved shear stress. The sum total of the displacement matrix as indicated in table 1 is symmetric signifying that there is no crystal rotation. The magnitudes of the tensile and the transverse strains are given by

$$
\begin{aligned}
& \varepsilon_{\langle 001\rangle}=\frac{8 \gamma}{\sqrt{6}}, \\
& \varepsilon_{\langle 010\rangle}=\varepsilon_{\langle 100\rangle}=\frac{-4 \gamma}{\sqrt{6}} .
\end{aligned}
$$

Since the magnitudes of transverse strain along two perpendicular directions are identical it may be concluded that the cross-sectional shape of the crystal remains unchanged.

However, the situation is quite different if the orientation of the tensile axis were more complex. For example, if it were [123] deformation on only one of the $12\{111\}$ $\langle 1 \overline{1} 0\rangle$ slip systems having maximum resolved shear stress will dominate its response to creep. In this case the preferred slip system is (111)[101]. Therefore the displacement, strain and rotation tensors are given by

$$
\begin{aligned}
e_{i j} & =\frac{\gamma}{\sqrt{6}}\left(\begin{array}{rrr}
-1 & -1 & -1 \\
0 & 0 & 0 \\
1 & 1 & 1
\end{array}\right), \\
\varepsilon_{i j} & =\frac{\gamma}{\sqrt{6}}\left(\begin{array}{ccl}
-1 & -1 / 2 & 0 \\
-1 / 2 & 0 & 1 / 2 \\
0 & 1 / 2 & 1
\end{array}\right), \\
\omega_{i j} & =\frac{\gamma}{\sqrt{6}}\left(\begin{array}{ccc}
0 & -1 / 2 & -1 \\
1 / 2 & 0 & -1 / 2 \\
1 & 1 / 2 & 0
\end{array}\right) .
\end{aligned}
$$


Table 1. Construction of displacement matrix of cubic single crystal with [001] as tensile axis and $\{111\}\langle 110\rangle$ as slip system.

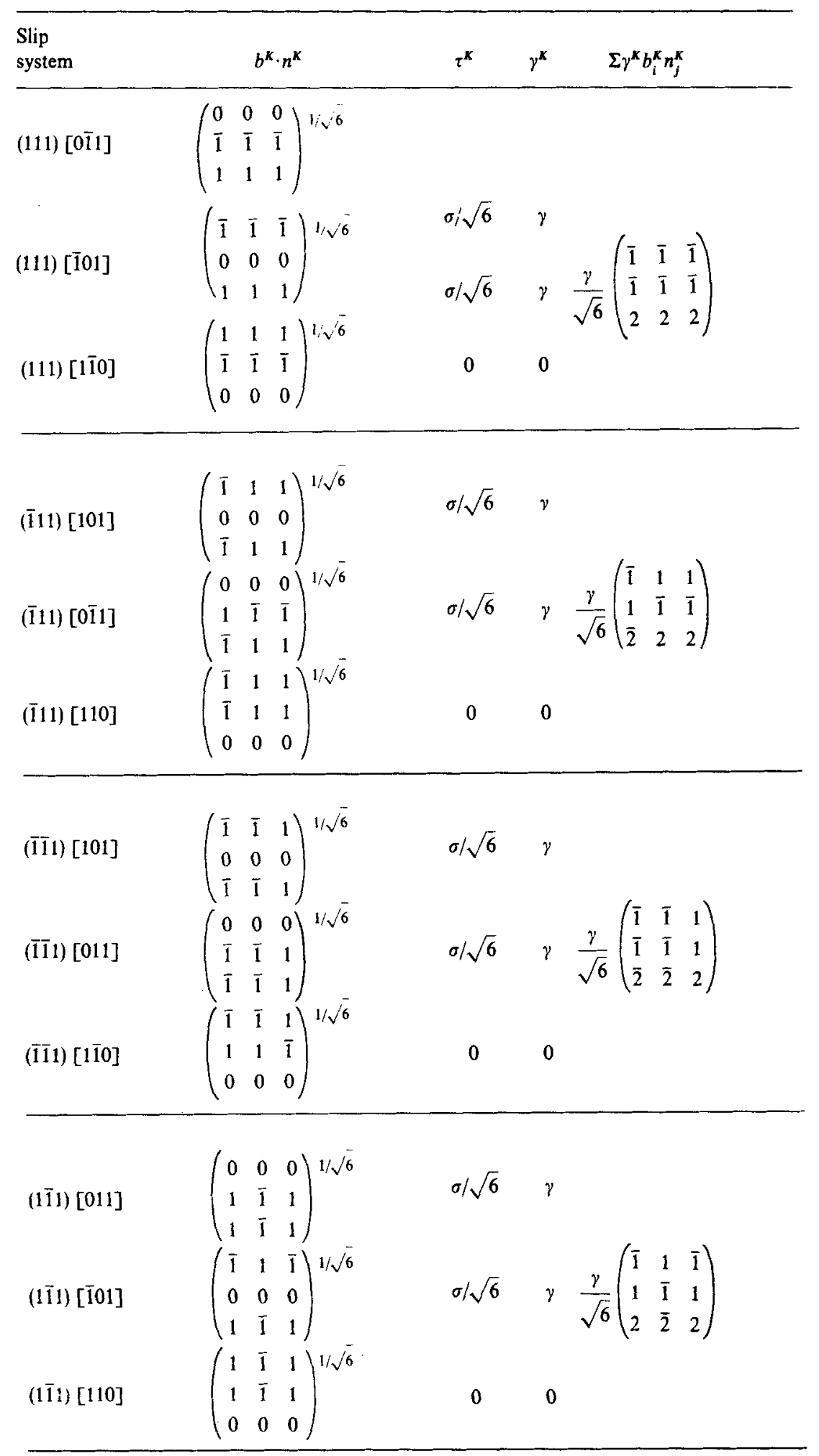

$$
\sum_{K=1}^{12} e_{i j}^{K}=\frac{\gamma}{\sqrt{6}}\left(\begin{array}{lll}
\overline{4} & 0 & 0 \\
0 & \overline{4} & 0 \\
0 & 0 & 8
\end{array}\right)
$$


Equations (4), (9) and (10) can be solved to obtain the axis as well as the magnitude of rotation. In this case these are $[1 \overline{2} 1]$ and $\gamma /(2 \sqrt{14})$ respectively. This means that the axis of rotation is perpendicular to the slip plane normal as well as the slip direction. Its magnitude is proportional to the accumulated shear strain. This has been represented on a stereographic projection in figure 3 . Following the same logic

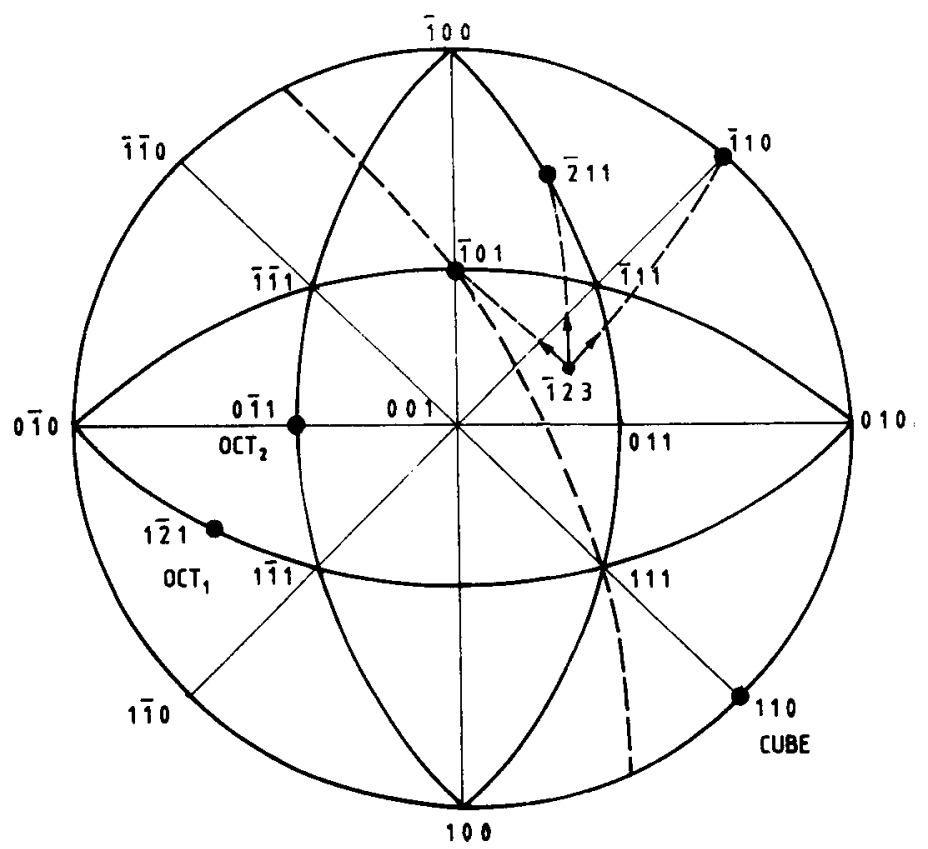

Figure 3. A stereographic projection illustrating the likely axis of rotation for [123] crystal

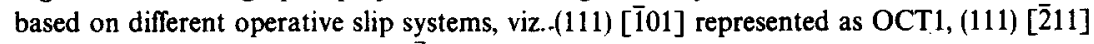
represented as OCT2, and (001) [110] represented as CUBE.

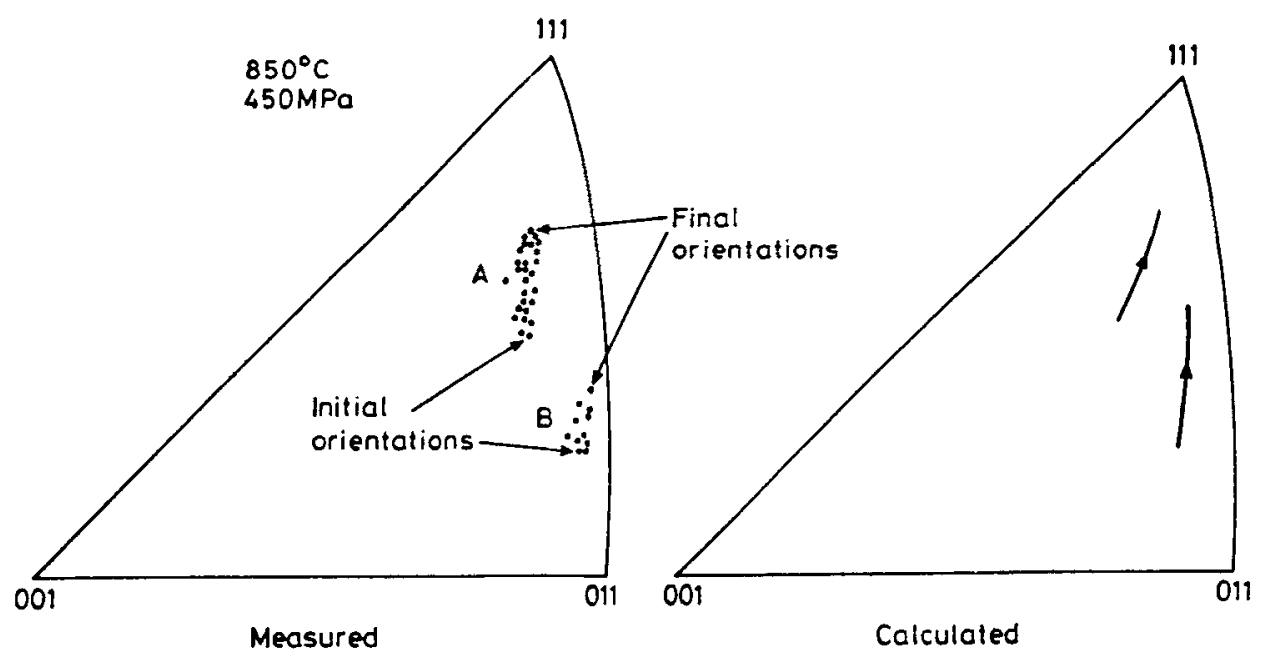

Figure 4. A comparison of observed and calculated crystal rotations during creep deformation of SRR99 of different complex orientations at $850^{\circ} \mathrm{C}$ and $450 \mathrm{MPa}$ assuming that both octahedral and cube slip are operative. 

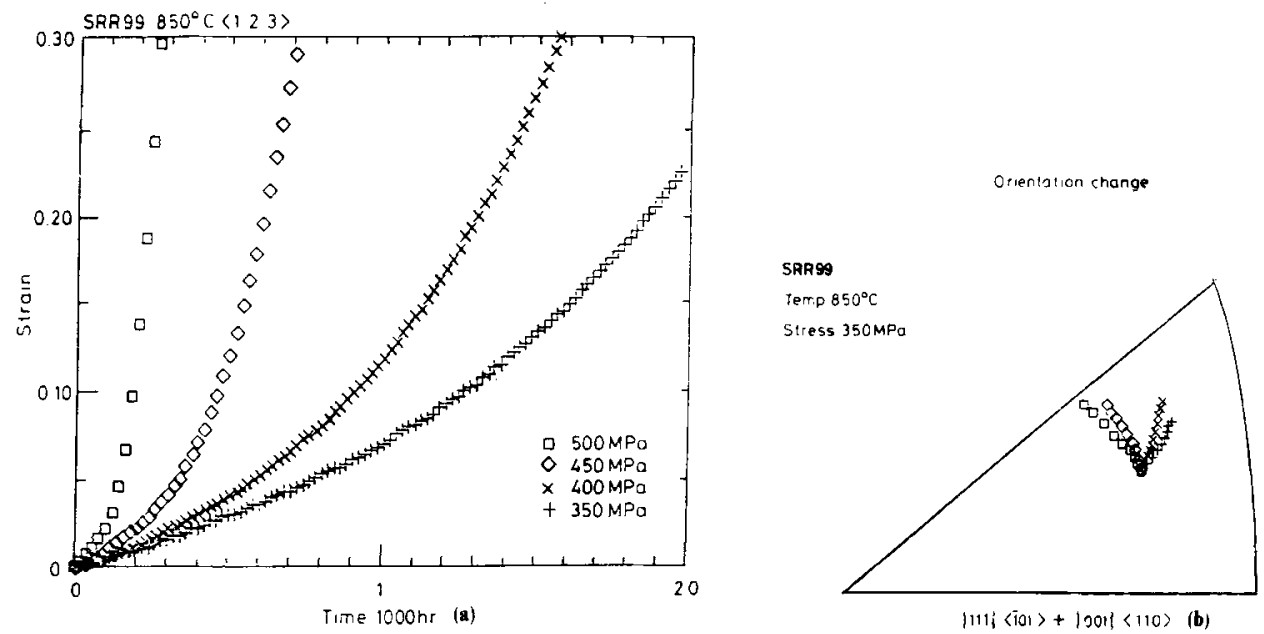

Figure 5. An illustration showing the effect of the applied stress on (a) creep strain accumulation and (b) crystal rotation for SRR99 crystal having $\langle 123\rangle$ as tensile axis at $850^{\circ} \mathrm{C}$.

the likely rotation path if the slip system were either (111) [101] or (001) [110] have been shown. Experimental data reported by McLean et al (1992) on SRR99 are shown in figure 4 . A comparison with figure 3 will indicate that such a rotation cannot be explained by only octahedral or cube slip. It is indeed more likely that shear deformation takes place simultaneously on both octahedral and cube planes. Using the material constants estimated by Ghosh et al (1990) creep curves for [123] crystals have been theoretically calculated at various levels of stress. These have been shown in figure 5a. The likely rotations have been indicated in figure $5 \mathrm{~b}$. This shows that the direction of movement of the tensile axis is a function of applied stress. A comparison with the rotation paths indicated in figure 3 . would reveal that at low stresses cube slip is more dominant whereas at higher stresses octahedral slip dominates. Therefore a model of creep deformation based on the concept of multiple slip on two types of slip system is capable of describing the observed nature of rotation. Using this concept the performance of SRR99 for all possible orientations can be determined. A summary of such calculations has been presented in figure 6 in the form of a performance map. This shows that at $850^{\circ} \mathrm{C} / 400 \mathrm{MPa},[001]$ crystal exhibits better creep properties, which is quite consistent with experimental data (Ghosh et al 1990). Such an exercise clearly illustrates usefulness of a model-based approach for characterizing the creep behaviour of anisotropic material. It can significantly cut down the volume of costly experimental work required to generate such maps. In addition it also suggests simple methods of determining the active slip system by measuring the change in cross-sectional shape of certain crystals of specific orientation. Figure 7a represents the progressive change in the shape of crystal having an initial tensile axis along [123] at a stress where creep deformation is dominated by cube slip; figure $7 \mathrm{~b}$ represents similar plots to show cross-sectional shape change when slip takes place primarily on octahedral planes. The difference in the predicted shape change clearly demonstrates the influence of slip system on shape change. This method appears to be quite simple and attractive in comparison to direct measurement of crystal orientation using back-reflection Laue X-ray diffraction pattern. Although 
such a method could estimate the orientation of crystal prior to testing with an accuracy of $1^{\circ}$, it has proved to be ineffective in case of deformed crystals. Diffraction patterns in these cases are too diffuse to allow meaningful orientations to be determined possibly because of accumulation of dislocations. Experimental data on crystal rotation reported by McLean et al (1992) were obtained from an analysis of

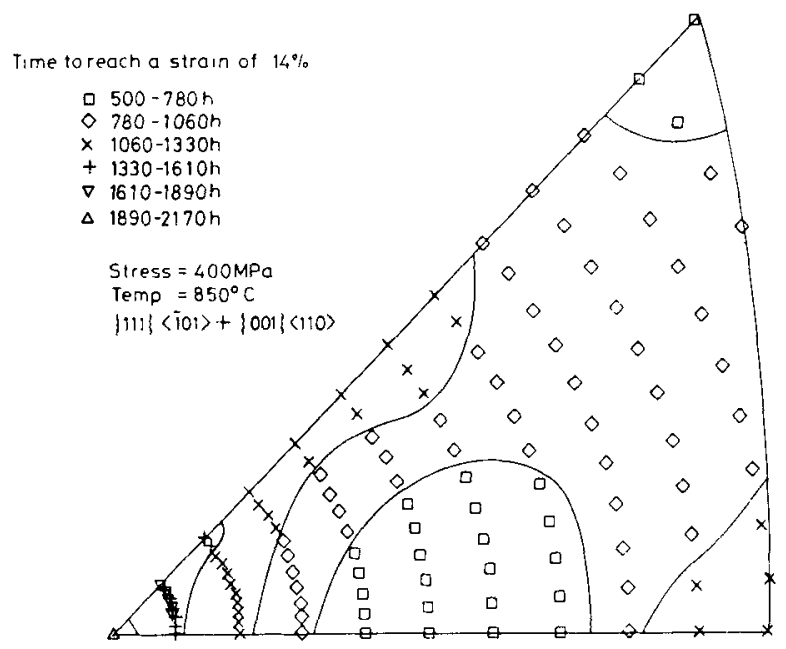

Figure 6. Computed anisotropy of creep performance for SRR 99 at $400 \mathrm{MPa} / 850^{\circ} \mathrm{C}$ displayed as contours in the unit stereographic triangle as time to reach $14 \%$ strain.
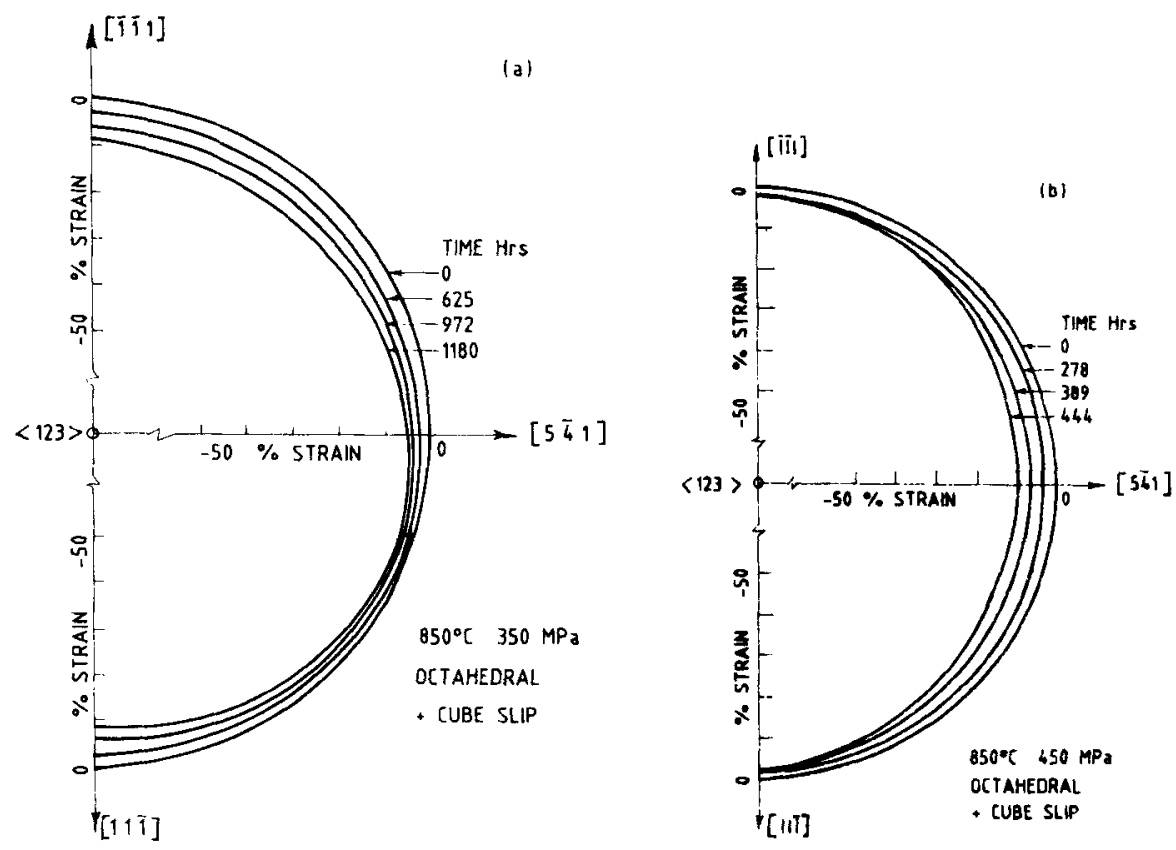

Figure 7. Progressive change in the cross-sectional shape of $\langle 123\rangle$ crystal at two different stresses: (a) at $350 \mathrm{MPa}$ cube slip is more active, and (b) at $450 \mathrm{MPa}$ octahedral slip is more active. 
electron backscatter patterns (EBSP) on a scanning electron microscope. Details of the method are described by Quested et al (1988).

Some of the experimental data on shape change reported by McLean et al (1992) do indicate that both cube and octahedral planes contribute to creep deformation in SRR99. However, such data are limited. Clearly there is a need to undertake more work in this direction. Nevertheless the present work does suggest a very simple method of establishing the type of operating slip system during creep in superalloys.

\section{Conclusions}

(i) A shear stress-based model has been developed for predicting the creep behaviour of a crystal of any arbitrary orientation.

(ii) Analysis of available data on crystal rotation using the model indicates that both octahedral and cube slips are operative in SRR99.

(iii) A simple method for identification of the operative slip system has been suggested. This is based on measurement of the change in cross-sectional shape of the specimen.

\section{Acknowledgement}

The author thanks Prof. Malcolm McLean, Department of Materials, Imperial College, London, for his guidance, encouragement and suggestions during the course of the work.

\section{References}

Caron P, Ohta Y, Nakagawa Y G and Khan T 1988 in Superalloys 1988 (ed.) D N Duhl et al (Warrendale, PA: The Metallurgical Society) p. 215

Curtis R V, Ghosh R N and McLean M 1993 in Proc. 5th Intl. Conf. on Creep and Fracture of Engg Materials and Structures (eds) B. Wilshire and R W Evans, University College, Swansea p. 149

Dyson B F 1988 Rev. Phys. Appl. 23605

Dyson B F and Ashby M F 1984 in Proc. 6th Intl. Conf. on Fracture (ICF 6), New Delhi (Oxford: Pergamon) p. 3

Dyson B F and Gibbons T B 1987 Acta Metall. 352355

Dyson B F and McLean M 1990 J. Iron Steel Inst. Jpn. 30802

Ghosh R N, Curtis R V and McLean M 1990 Acta Metall. Mater. 381977

Kachanov L M $1958 \mathrm{Izv}$. Akad. Nauk. SSSR No. 8, 26

Kear B H and Piearcey B J 1967 Trans. Metall. Soc. AIME 2391209

Leckie F A and Hayhurst D R 1977 Acta Metall. 251959

McLean M, Ghosh R N, Curtis R V, Basu Conlin U and Winstone M R 1992 in Superalloys 1992 (eds)

S D Antolovich et al (Warrendale, PA: The Metallurgical Society) p. 609

Quested P N, Handerson P J and McLean M 1988 Acta Metall. 362743

Robonotov Yu N 1969 in Proc. XII IU TAM Congress (eds) H Hetengi and W G Vincenti(Berlin: Springer) 